\title{
Spectra of principal graphs
}

Vijay Kodiyalam and V.S. Sunder

The Institute of Mathematical Sciences

Chennai 600113, INDIA

e-mail: vijay@imsc.ernet.in , sunder@imsc.ernet.in

August 22, 2007 


\begin{abstract}
We show that if the adjacency matrices of the two principal graphs of a finite index subfactor are regarded as (necessarily bounded, self-adjoint) operators on the $\ell^{2}$ spaces over their vertex sets, then their spectral measures, when restricted to the complement of $\{0\}$, are mutually absolutely continuous. In particular, for a finite-depth subfactor, the two matrices have the same sets of non-zero eigenvalues.
\end{abstract}




\section{Introduction}

We gather together the notation and terminology that we will need, in order to prove the result stated in the abstract. In the sequel, we shall follow standard notation from subfactor theory - as in [JS], say.

Suppose $R_{0} \subset R_{1}$ is a subfactor of finite Jones index; i.e., this is an inclusion of $I I_{1}$ factors such that $\operatorname{dim} R_{0} L^{2}\left(R_{1}\right)<$ $\infty$. For $i=0,1$, let us write $\alpha_{i, 1-i}$ to denote the bimodule ${ }_{R_{i}} L^{2}\left(R_{1}\right)_{R_{1-i}}$ which has finite left- and right- dimensions under the assumption of finite index. (In fact, this can be taken as the definition of finiteness of index.) Then the so-called contragredient bimodule of $\alpha_{01}$ is nothing but $\alpha_{10}$; i.e., $\overline{\alpha_{01}}=\alpha_{10}$.

For $i, j \in\{0,1\}$, let us write $B_{i j}$ for the set of isomorphism classes of irreducible $R_{i}-R_{j}$ bimodules contained in tensor products of the form $\alpha_{i, 1-i} \otimes_{R_{1-i}} \alpha_{1-i, i} \otimes_{R_{i}} \cdots \otimes_{R_{1-j}} \alpha_{1-j, j}$, where the above string of bimodules must start with $\alpha_{i, 1-i}$ and end with $\alpha_{1-j, j}$, but is allowed to be arbitrarily long provided its length is of the same parity as $(j-i)$.

The so-called pair of principal graphs of the subfactor is $\left(\Gamma_{0}, \Gamma_{1}\right)$, where, for $p=0,1$, the graph $\Gamma_{p}$ is the bipartite graph with vertex set $B_{0 p} \coprod B_{1 p}$, with the number of edges joining $\gamma_{0 p}$ and $\gamma_{1 p}$ being given by the multiplicity $\left\langle\alpha_{10} \otimes_{R_{0}} \gamma_{0 p}, \gamma_{1 p}\right\rangle$ with which the bimodule $\gamma_{1 p}$ features in the tensor product $\alpha_{10} \otimes_{R_{0}} \gamma_{0 p}$. (Above, and in the sequel, we shall use the symbol Ц to indicate 'disjoint union'. And we shall adopt the 'subscript convention' whereby a symbol such as $\gamma_{i j}$ will always denote an element of $B_{i j}$; the only possible exception to this rule stems from the possibility that in case our subfactor is not irreducible, then $\alpha_{i, 1-i}$ is not one element in $B_{i, 1-i}$, but a direct sum of finitely many members of $B_{i, 1-i}$. Further, all subscripts, such as $i, j, p$ will run over the set $\{0,1\}$.)

To any graph $\Gamma$, with vertex set $V=V(\Gamma)$, is associated its adjacency matrix $A=A(\Gamma)$, which has rows and columns indexed by $V(\Gamma)$, and is the symmetric matrix (with non-negative integral entries), given by $A(u, v)$ equal to the number of edges joining $u$ and $v$.

The subfactor $R_{0} \subset R_{1}$ is said to have finite depth precisely 
when each $B_{i j}$ is a finite set, i.e, if the graphs $\Gamma_{p}$ are both finite. (In fact, the finiteness of either one of them implies that of the other.) Even in the infinite-depth case, it is true - see Remark 2.2(c) - that the matrix $A_{p}=A\left(\Gamma_{p}\right)$ defines a bounded (self-adjoint) operator on $\mathcal{L}\left(\ell^{2}\left(V\left(\Gamma_{p}\right)\right)\right)$.

Henceforth the symbol $\Delta$ will denote a Borel set in $\mathbb{R}$ such that $0 \notin \Delta$.

Definition 1.1 If $A$ and $B$ are self-adjoint operators on Hilbert space, we shall write $A \sim_{0} B$ if the following condition is satisfied:

$$
1_{\Delta}(A) \neq 0 \Leftrightarrow 1_{\Delta}(B) \neq 0 \quad \forall \Delta .
$$

Above, and elsewhere, the symbol $1_{\Delta}(A)$ denotes the spectral projection for the self-adjoint operator corresponding to the set $\Delta$; the notation is natural, in the sense of the measurable functional calculus for self-adjoint operators. It should be obvious that $\sim_{0}$ is an equivalence relation. Notice that if the definition above is relaxed to only considering open sets not containing 0 , then the corresponding equivalence relation would just be: $s p(A) \backslash\{0\}=s p(B) \backslash\{0\}$, where the symbol $s p$ denotes 'spectrum'.

This paper is devoted to the proof of the following theorem:

THEOREM 1.2 With the foregoing notation, we have:

$$
A_{0} \sim_{0} A_{1} .
$$

Some immediate consequences of this theorem are listed in the following corollary, whose proof is disscussed after the proof of the theorem.

Corollary 1.3 With $A_{0}$ and $A_{1}$ as above, we have:

(i) with the only possible exception of 0 , the operators $A_{p}$ have the same spectrum;

(ii) when restricted to $\mathbb{R} \backslash\{0\}$, the spectral measures of the $A_{p}$ 's are mutually absolutely continuous;

(iii) with the only possible exception of 0 and isolated nonzero eigenvalues of infinite multplicity, the operators $A_{p}$ have the same essential spectrum. 
Finally, in the case of Haagerup's $\frac{5+\sqrt{13}}{2}$ subfactor - see [H] and $[\mathrm{AH}]$ - both the numbers $\pm \sqrt{2}$ are eigenvalues of multiplicity 1 for one of the operators and multiplicity 2 for the other, thereby showing that the two self-adjoint operators can have different spectral multiplicity functions.

\section{Proof of the main result}

The possibility of forming tensor-products and contragredients of bimodules endows the structure of an an involutive algebra on the $\mathbb{C}$-vector space $\mathcal{A}$ with basis given by $\coprod_{i, j=0,1} B_{i j}$. We shall need this $M_{2}$-graded fusion algebra in what follows, so we spell out the desired facts as a proposition for convenience of future reference. We omit the proof of this well-known fact; the interested reader might wish to consult [EK], for instance.

Proposition 2.1 Let $\mathcal{A}$ be the $\mathbb{C}$-vector space with basis $\coprod_{i, j=0,1} B_{i j}$. Define

$$
\gamma_{i j} \gamma_{k l}=\left\{\begin{array}{cl}
0 & \text { if } j \neq k \\
\sum_{\gamma_{i l}}\left\langle\gamma_{i j} \otimes_{R_{j}} \gamma_{j l}, \gamma_{i l}\right\rangle \gamma_{i l} & \text { if } j=k
\end{array}\right.
$$

and define $\overline{\gamma_{i j}}$ to be that unique $\gamma_{j i} \in B_{j i}$ which is isomorphic to the contragredient of the bimodule $\gamma_{i j}$. Then,

(i) $\mathcal{A}$ is an associative involutive algebra;

(ii) $\left\langle\gamma_{i j} \otimes_{R_{j}} \gamma_{j l}, \gamma_{i l}\right\rangle=\left\langle\overline{\gamma_{i j}} \otimes_{R_{i}} \gamma_{i l}, \gamma_{j l}\right\rangle$; and

(iii) there exist elements $1_{i} \in B_{i i}, i=0,1$, such that

$$
1_{i} \gamma_{i j}=\gamma_{i j}=\gamma_{i j} 1_{j} \forall \gamma_{i j}
$$

and in particular, the element $1=1_{0}+1_{1}$ is a multiplicative identity for $\mathcal{A}$.

When convenient, we shall (i) write $\mathcal{B}=\coprod_{i, j=0,1} B_{i j}$; (ii) denote the typical elements of $\mathcal{B}$ by the symbols $X, Y, Z$; and (iii) for $X, Y, Z \in \mathcal{B}$, write $N_{X Y}^{Z}$ for the 'fusion coefficients' defined by the equation $X Y=\sum_{Z \in \mathcal{B}} N_{X Y}^{Z} Z$ (in $\mathcal{A}$ ).

We first list some easy consequences of Proposition 2.1. 
REMARK 2.2 (a)

$$
\sum_{p=0}^{1} N_{X Y}^{1_{p}}=\sum_{p=0}^{1} N_{\bar{X} 1_{p}}^{Y}=\delta_{\bar{X}, Y}, \forall X, Y \in \mathcal{B}
$$

(b) We shall write $\mathcal{A} \ni x \mapsto L_{x} \in \operatorname{End}_{\mathbb{C}}(\mathcal{A})$ to denote the left regular representation (defined by $L_{x}(y)=x y$ ). The map $L$ is one-to-one since $\mathcal{A}$ has an identity.

Equip $\mathcal{A}$ with an inner product so that $\mathcal{B}$ is an orthonormal set. Then the Hilbert space completion of $\mathcal{A}$ with respect to this inner product is naturally identified with the space $\ell^{2}(\mathcal{B})$ of square-summable functions on the set $\mathcal{B}$. We shall write $\mathcal{H}_{i j}$ for the Hilbert subspace which has $B_{i j}$ as an orthonormal basis.

Notice that $\operatorname{End}_{\mathbb{C}}(\mathcal{A})$ is naturally identified with "columnfinite' matrices (with rows and columns indexed by $\mathcal{B}$ ) - whereby $T \in \operatorname{End}_{\mathbb{C}}(\mathcal{A})$ corresponds to the matrix $\left(\left(t_{Y Z}\right)\right)_{Y, Z \in \mathcal{B}}$ given by $t_{Y Z}=\langle T(Z), Y\rangle \forall Y, Z \in \mathcal{B}$.

(c) Observe that if $X \in \mathcal{B}$, then

$$
\begin{aligned}
\left(L_{\bar{X}}\right)_{Y Z} & =\langle\bar{X} Z, Y\rangle \\
& =N_{\bar{X} Z}^{Y} \\
& =N_{X Y}^{Z} \\
& =\langle X Y, Z\rangle \\
& =\left(L_{X}\right)_{Z Y}
\end{aligned}
$$

Thus if $X \in \mathcal{B}$, then the matrix of $L_{\bar{X}}$ is the transpose of the matrix of $L_{X}$; and so, if $x \in \mathcal{A}$, it follows that the matrix of $L_{\bar{x}}$ is the adjoint (= conjugate-transpose) of the matrix of $L_{x}$. In particular, each $L_{x}, x \in \mathcal{A}$ is represented by a matrix which is both row-finite and column-finite.

We now outline a proof of the fact that $L_{x}$ extends uniquely to a bounded operator, for each $x \in \mathcal{A}$. It suffices to check this when $x \in \mathcal{B}$. So, suppose $x=\gamma \in \mathcal{B}_{p q}$. A little thought reveals that it suffices to verify that the matrix - call it $T$ - of $L_{\gamma \bar{\gamma}}$ represents a bounded operator. Notice that $\gamma \bar{\gamma} \in \mathcal{H}_{p p}$ and that the matrix of $T$ satisfies: $t_{Y Z}=0$ unless both $Y$ and $Z$ belong to $B_{p j}$ for $j=0,1$. So, it suffices to check that if $T_{j}$ is 
the (principal) submatrix of $T$ obtained by restricting the rows and columns to $B_{p j}$, then $T_{j}$ is bounded for $j=0,1$. Now, it is a fact (see [JS], for instance) that if $P, Q$ are $I I_{1}$ factors, if $\mathcal{H}_{1}$ is a $P-Q$ bimodule and $\mathcal{H}_{2}$ is a left $Q$-module, then $\operatorname{dim}_{P}\left(\mathcal{H}_{1} \otimes_{Q} \mathcal{H}_{2}\right)=\operatorname{dim}_{P}\left(\mathcal{H}_{1}\right) \operatorname{dim}_{Q}\left(\mathcal{H}_{2}\right)$. It follows quite easily from this that if we define the vector $v_{j}$ by $v_{j}(Y)=\operatorname{dim}_{R_{p}}(Y)$ for $Y \in B_{p j}$, then $v_{j}$ is a vector with strictly positive entries (which is not in $\ell^{2}\left(B_{p j}\right)$ if $B_{p j}$ is infinite) such that $T v_{j}=\lambda v_{j}$, where $\lambda=\operatorname{dim}_{R_{p}}\left(\gamma \otimes_{R_{q}} \bar{\gamma}\right)$. However, a symmetric matrix with a strictly positive eigenvector is known - see $[\mathrm{P}]$ - to define a bounded operator on the $\ell^{2}$ space, with norm at most equal to the eigenvalue $\lambda$. (A formulation of a more general statement, called the 'Schur test', may be found in [HS].)

Thus, we see that $x \mapsto L_{x}$ defines a ${ }^{*}$-homomorphism from $\mathcal{A}$ into $\mathcal{L}\left(\ell^{2}(\mathcal{B})\right)$.

(d) Let $M=\left(\left\{L_{x}: x \in \mathcal{A}\right\}\right)^{\prime \prime}$ denote the von Neumann algebra completion of $\mathcal{A}$ (in $\mathcal{L}\left(\ell^{2}(\mathcal{B})\right)$ ). It is clear that 1 is a cyclic vector for $M$, since $M 1 \supset \mathcal{B}$. On the other hand, we may argue exactly as in (c) that for each $X \in \mathcal{B}$, there exists a unique operator $R_{X} \in \mathcal{L}\left(\ell^{2}(\mathcal{B})\right)$ such that $R_{X}(Y)=Y X$ for all $X \in \mathcal{B}$. It should be obvious that $R_{X} \in\left(\left\{L_{x}: x \in \mathcal{A}\right\}\right)^{\prime}=M^{\prime}$; and that hence the vector 1 is also a cyclic vector for $M^{\prime}$; consequently, (see [JS], for instance) we see that 1 is a cyclic and separating vector for $M$. Hence the functional defined by

$$
M \ni x \mapsto \tau(x)=\langle x(1), 1\rangle
$$

is seen to be a faithful positive normal linear functional. Actually, it turns out that $\frac{1}{2} \tau$ is a faithful normal tracial state on $M$, but we will not need this, and shall say no more about it.

In what follows, if $V_{0}, V_{1}$ are sets and if a $V_{0} \times V_{1}$ matrix, say $G$, represents a bounded operator from $\ell^{2}\left(V_{1}\right)$ to $\ell^{2}\left(V_{0}\right)$, then, we will use the same symbol to represent this operator as well. So, the symbol $G^{*}$ will represent the conjugate-transpose matrix as well the adjoint operator of $G$.

Lemma 2.3 Suppose $\Gamma$ is a bipartite graph, with the associated partition of its vertex set being given by $V=V_{0} \amalg V_{1}$. Then, 
with respect to this decomposition of the vertex set, the adjacency matrix $A=A(\Gamma)$ clearly has the decomposition

$$
A=\left[\begin{array}{cc}
0 & G \\
G^{*} & 0
\end{array}\right],
$$

where $G$ is the $V_{0} \times V_{1}$ matrix defined by setting $G\left(v_{0}, v_{1}\right)$ equal to the number of edges joining $v_{0}$ and $v_{1}$. Assume that $A$ induces a bounded (necessarily self-adjoint) operator on $\ell^{2}(V)$.

For any $0 \notin \Delta \in \mathcal{B}_{\mathbb{R}}$, we have:

$$
1_{\Delta}(A) \neq 0 \Leftrightarrow 1_{\Delta^{2}}\left(G G^{*}\right) \neq 0 \Leftrightarrow 1_{\Delta^{2}}\left(G^{*} G\right) \neq 0,
$$

where we write $\Delta^{2}$ for $\left\{\lambda^{2}: \lambda \in \Delta\right\}$.

Proof: To start with, if $T=W\left(T^{*} T\right)^{\frac{1}{2}}$ is the (left) polar decomposition of a bounded operator $T$ between Hilbert spaces, it is then true that (a) the initial and final spaces of the partial isometry $W$ - call them $\mathcal{M}$ and $\mathcal{N}$ - satisfy $\mathcal{M}=$ $\operatorname{ran}\left(1_{\mathbb{R} \backslash\{0\}}\left(T^{*} T\right)\right)$ and $\mathcal{N}=\operatorname{ran}\left(1_{\mathbb{R} \backslash\{0\}}\left(T T^{*}\right)\right)$, respectively; and (b) when regarded as a unitary operator of $\mathcal{M}$ onto $\mathcal{N}$, the operator $\left.W\right|_{\mathcal{M}}$ establishes a unitary equivalence between the operators $\left.\left(T^{*} T\right)\right|_{\mathcal{M}}$ and $\left.\left(T T^{*}\right)\right|_{\mathcal{N}}$. In particular, it follows that for every $\Delta \in \mathcal{B}_{\mathbb{R}}$,

$$
1_{\Delta}\left(G^{*} G\right) \neq 0 \Leftrightarrow 1_{\Delta}\left(G G^{*}\right) \neq 0 .
$$

The next fact we need is that if $T$ is a self-adjoint operator on a Hilbert space, if $\Delta \subset(0, \infty)$, and if we write $-\Delta=\{-\lambda$ : $\lambda \in \Delta\}$, then

$$
1_{-\Delta \amalg \Delta}(T)=1_{\Delta^{2}}\left(T^{2}\right) .
$$

(This is a simple consequence of the spectral theorem and a 'change of variable' formula in measure theory, whose proof we omit.)

Observe next that if

$$
U=\left[\begin{array}{cc}
i d_{\ell^{2}\left(V_{0}\right)} & 0 \\
0 & -i d_{\ell^{2}\left(V_{1}\right)}
\end{array}\right]
$$

then $U$ is unitary and $U A U^{*}=-A$. Hence $A$ and $-A$ are unitarily equivalent, and in particular,

$$
1_{\Delta}(A) \neq 0 \Leftrightarrow 1_{-\Delta}(A) \neq 0 \Leftrightarrow 1_{-\Delta \cup \Delta}(A) \neq 0 .
$$


Let us write $\mathbb{R}^{+}=[0, \infty), \mathbb{R}^{-}=(-\infty, 0]$; and let $\Delta_{ \pm}=$ $\Delta \cap \mathbb{R}^{ \pm}$; then $\Delta=\Delta_{+} \amalg \Delta_{-}$and $\Delta^{2}=\Delta_{+}^{2} \cup \Delta_{-}^{2}$; we see therefore that

$$
1_{\Delta^{2}}(T) \leq 1_{\Delta_{+}^{2}}(T)+1_{\Delta_{-}^{2}}(T) \leq 21_{\Delta^{2}}(T)
$$

for any self-adjoint operator $T$.

Finally conclude that

$$
\begin{aligned}
& 1_{\Delta}(A) \neq 0 \Leftrightarrow 1_{\Delta_{+}}(A)+1_{\Delta_{-}}(A) \neq 0 \\
& \Leftrightarrow 1_{\Delta_{+}}(A) \neq 0 \text { or } 1_{\Delta_{-}}(A) \neq 0 \\
& \Leftrightarrow 1_{-\Delta_{+} \amalg \Delta_{+}}(A) \neq 0 \text { or } 1_{-\Delta_{-} \amalg \Delta_{-}}(A) \neq 0 \text { (by }(2.5) \text { ) } \\
& \Leftrightarrow 1_{\Delta_{+}^{2}}\left(A^{2}\right) \neq 0 \text { or } 1_{\Delta_{-}^{2}}\left(A^{2}\right) \neq 0 \text { (by }(2.4) \text { ) } \\
& \Leftrightarrow 1_{\Delta_{+}^{2}}\left(A^{2}\right)+1_{\Delta_{-}^{2}}\left(A^{2}\right) \neq 0 \\
& \Leftrightarrow 1_{\Delta^{2}}\left(A^{2}\right) \neq 0 \text { (by }(2.6) \text { ) } \\
& \Leftrightarrow 1_{\Delta^{2}}\left(\left[\begin{array}{cc}
G G^{*} & 0 \\
0 & G^{*} G
\end{array}\right]\right) \neq 0 \\
& \Leftrightarrow\left[\begin{array}{cc}
1_{\Delta^{2}}\left(G G^{*}\right) & 0 \\
0 & 1_{\Delta^{2}}\left(G^{*} G\right)
\end{array}\right] \neq 0 ;
\end{aligned}
$$

and the desired conclusion follows now from equation (2.3) and the above implications.

Proof of Theorem 1.2: For convenience, let us simply write $\alpha$ for what we denoted earlier by $\alpha_{01}$; then $\bar{\alpha}$ is what we earlier denoted by $\alpha_{10}$; further, for $p=0,1$, if we write $A_{p}$ for the $\left(B_{0 p} \amalg B_{1 p}\right) \times\left(B_{0 p} \amalg B_{1 p}\right)$ matrix and $G_{p}$ for the $B_{0 p} \times B_{1 p}$ matrix which are associated to the bipartite graph $\Gamma_{p}$ as in Lemma 2.3 , we see that

$$
\begin{aligned}
G_{p}\left(\gamma_{0 p}, \gamma_{1 p}\right) & =\left\langle\bar{\alpha} \otimes_{R_{0}} \gamma_{0 p}, \gamma_{1 p}\right\rangle \\
& =\left\langle\alpha \otimes_{R_{0}} \gamma_{1 p}, \gamma_{0 p}\right\rangle \\
& =\left\langle L_{\alpha}\left(\gamma_{1 p}\right), \gamma_{0 p}\right\rangle
\end{aligned}
$$

and so $G_{p}$ is just the restricted operator $\left.L_{\alpha}\right|_{\mathcal{H}_{1 p}}: \mathcal{H}_{1 p} \rightarrow \mathcal{H}_{0 p}$; and so $G_{p}^{*}=\left.L_{\bar{\alpha}}\right|_{\mathcal{H}_{0 p}}: \mathcal{H}_{0 p} \rightarrow \mathcal{H}_{1 p}$. Hence, we deduce from Lemma 2.3 that

$$
1_{\Delta}\left(A_{p}\right) \neq 0 \Leftrightarrow 1_{\Delta^{2}}\left(\left.L_{\bar{\alpha} \alpha}\right|_{\mathcal{H}_{1 p}}\right) \neq 0 \Leftrightarrow 1_{\Delta^{2}}\left(\left.L_{\alpha \bar{\alpha}}\right|_{\mathcal{H}_{0 p}}\right) \neq 0 .
$$


Notice next that $L_{\bar{\alpha} \alpha}=L_{\bar{\alpha}} L_{\alpha}$ leaves each of the subspaces $\mathcal{H}_{10}, \mathcal{H}_{11}$ invariant, and is identically 0 on $\mathcal{H}_{00} \oplus \mathcal{H}_{01}$. Thus if we let $P_{i j}$ denote the orthogonal projection onto $\mathcal{H}_{i j}$, then $P_{00}+P_{01} \leq 1_{\{0\}}\left(L_{\bar{\alpha} \alpha}\right)$.

We shall now prove that

$$
\left.\left.L_{\bar{\alpha} \alpha}\right|_{\mathcal{H}_{11} \oplus \mathcal{H}_{10}} \sim_{0} \quad L_{\bar{\alpha} \alpha}\right|_{\mathcal{H}_{11}} .
$$

For typographical convenience, let us write $T=\left.L_{\bar{\alpha} \alpha}\right|_{\mathcal{H}_{11} \oplus \mathcal{H}_{10}}$, and $T_{1}=\left.T\right|_{\mathcal{H}_{11}}$. Thus, we need to show that, for all $\Delta$,

$$
1_{\Delta}(T) \neq 0 \Leftrightarrow 1_{\Delta}\left(T_{1}\right) \neq 0 \text {. }
$$

Fix $\Delta \in \mathcal{B}_{\mathbb{R}}$ such that $0 \notin \Delta$. Since $1_{\Delta}\left(T_{1}\right)=\left.\left(P_{11} 1_{\Delta}(T)\right)\right|_{\mathcal{H}_{11}}=$ $\left.\left(1_{\Delta}(T) P_{11}\right)\right|_{\mathcal{H}_{11}}$, we always have:

$$
1_{\Delta}\left(T_{1}\right) \neq 0 \Rightarrow 1_{\Delta}(T) \neq 0 .
$$

So we need to verify that if $Q=1_{\Delta}(T) \neq 0$, then $Q P_{11} \neq 0$, ; we shall show that $Q\left(1_{1}\right) \neq 0$. For this, notice first that

$$
0 \notin \Delta \Rightarrow \operatorname{ran}\left(1_{\Delta}\left(L_{\bar{\alpha} \alpha}\right)\right) \subset \mathcal{H}_{11} \oplus \mathcal{H}_{10}
$$

and hence $1_{\Delta}\left(L_{\bar{\alpha} \alpha}\right)=Q$; further, since $1=1_{0}+1_{1}$ is a separating vector for $M$ - see Remark $2.2(\mathrm{~d})$ - and since $1_{0} \in \mathcal{H}_{00} \subset$ $\operatorname{ker}\left(L_{\bar{\alpha} \alpha}\right) \subset \operatorname{ker}\left(1_{\Delta}\left(L_{\bar{\alpha} \alpha}\right)\right)$, we find that

$$
Q\left(1_{1}\right)=1_{\Delta}\left(L_{\bar{\alpha} \alpha}\right)\left(1_{1}+1_{0}\right)=1_{\Delta}\left(L_{\bar{\alpha} \alpha}\right)(1) \neq 0,
$$

and equation (2.8) is verified.

On the other hand, since we clearly have

$$
1_{\Delta}(T)=1_{\Delta}\left(\left.T\right|_{\mathcal{H}_{10}}\right) \oplus 1_{\Delta}\left(\left.T\right|_{\mathcal{H}_{11}}\right),
$$

we see that

$$
\begin{aligned}
1_{\Delta}\left(A_{0}\right) \neq 0 & \Rightarrow 1_{\Delta^{2}}\left(\left.T\right|_{\mathcal{H}_{10}}\right) \neq 0 \quad(\text { by }(2.7)) \\
& \Rightarrow 1_{\Delta^{2}}\left(\left.T\right|_{\mathcal{H}_{11}}\right) \neq 0 \quad(\text { by }(2.9) \text { and }(2.8)) \\
& \Rightarrow 1_{\Delta}\left(A_{1}\right) \neq 0 \quad(\text { by }(2.7)) .
\end{aligned}
$$

The reverse implication is proved by exactly analogous reasoning applied with $\alpha \bar{\alpha}$ in place of $\bar{\alpha} \alpha$ (or, by applying the already proved implication to the dual subfactor). 
Proof of Corollary 1.3: The proof of (i) has already been outlined in the paragraph following Definition 1.1, while (ii) is just the verbal translation of the statement that $A_{0} \sim_{0} A_{1}$.

For (iii), first observe that if $T$ is a self-adjoint operator, and if $\lambda \in \mathbb{R}$, then $\lambda \in s p_{e}(T)$ - where we write ' $s p_{e}$ ' for the essential spectrum - if and only if $1_{\Delta}(T)$ has infinite rank for every open neighbourhood $\Delta$ of $\lambda$. From this, we see that for any $\lambda \in \mathbb{R}$, the following conditions are equivalent:

(a) $\lambda \in s p_{e}(T)$ and $\lambda$ is not an isolated eigenvalue of infinite multipilicity;

(b) every neighbourhood of $\lambda$ contains infinitely many points of $\operatorname{sp}(T)$;

(c) for every open neighbourhood $\Delta$ of $\lambda$, there exists an infinite collection $\left\{\Delta_{n}\right\}$ of pairwise disjoint open subsets of $\Delta$ such that $1_{\Delta_{n}}(T) \neq 0$ for all $n$.

It follows that if $A, B$ are self-adjoint operators and if $A \sim_{0} B$, and if $\lambda \in \mathbb{R}$, then $\lambda$ satisfies condition (c) and hence (a) above for $A$ if and only if it satisfies condition (c) and hence (a) for $B$.

The fact that the $A_{p}$ 's have the same norm has been known for a while. For related results (such as the fact that they even have the same essential norm), see [P1]. We conclude with a question suggested by Popa, which we formally state in the following 'conjecture'.

Conjecture: Let $A$ denote the adjacency matrix of the principal graph of a finite index subfactor. Then, $s p(A)=s p_{e}(A)$ in the infinite-depth case. 


\section{References:}

[AH] M. Asaeda and U. Haagerup, Exotic subfactors of finite depth with Jones indices $(5+\sqrt{13}) / 2$ and $(5+\sqrt{17}) / 2$, Commun. Math. Phys., 202, (1999), 1-63.

[EK] D.E. Evans, and Y. Kawahigashi, Quantum symmetry on operator algebras, Oxford University Press, 1998.

[H] U. Haagerup, Principal graphs of subfactors in the index range $4<[M: N]<3+\sqrt{3}$, Subfactors - Proc. of the Taniguchi Symp., Katata, World-Scientific, pp. 1-38, 1994.

[HS] P.R. Halmos and V.S. Sunder, Bounded Integral operators on $L^{2}$-spaces, Ergeb. der Math, 96, Springer-Verlag, Berlin, 1978.

[JS] V. Jones and V.S. Sunder, Introduction to subfactors, London Math. Soc. Lecture Note Series, 234, Cambridge University Press, Cambridge, 1997.

[P] S. Popa, Classification of amenable subfactors of type II, Acta Math., 172, (1994), 163-255.

[P1] S. Popa, Some ergodic properties of infinite graphs associated to subfactors, Ergodic Theory and Dyn. Sys., 15, 1995, 993-1003. 